\title{
Genomic Heterogeneity among French Rhizobium Strains Isolated from Phaseolus vulgaris L.
}

\author{
G. LAGUERRE, ${ }^{1 *}$ M. P. FERNANDEZ, ${ }^{2}$ V. EDEL,${ }^{1 \dagger}$ P. NORMAND, ${ }^{2}$ AND N. AMARGER ${ }^{1}$ \\ Laboratoire de Microbiologie des Sols, Institut National de la Recherche Agronomique, 17 rue Sully \\ BV. 1540, F-21034 Dijon Cedex, ${ }^{1}$ and Laboratoire d'Ecologie Microbienne et Centre d'Etude Moléculaire \\ de la Biodiversité, Université Claude Bernard Lyon I, F-69622 Villeurbanne Cedex, ${ }^{2}$ France
}

\begin{abstract}
Levels of DNA relatedness between strains isolated from root nodules of Phaseolus vulgaris and reference strains of different Rhizobium species were determined by performing DNA-DNA hybridization experiments (S1 nuclease method). The nine strains examined were members of three genomic groups previously delineated by a restriction fragment length polymorphism analysis among strains isolated from $P$. vulgaris at different sites in France. In agreement with the results of the restriction fragment length polymorphism analysis, three genomic species were found. We confirmed that one of these species corresponded to Rhizobium leguminosarum since the strain examined was $100 \%$ related to the type strain of this species. The other two species were new genomic species which were less than $21 \%$ related to reference strains belonging to other Rhizobium species, including Rhizobium etli and Rhizobium tropici, and were $18 \%$ related to each other. As determined by an analysis of partial $16 \mathrm{~S}$ ribosomal DNA sequences, each of the genomic species was found to belong to a lineage independent from the lineages of previously described Rhizobium species. Nevertheless, they were included in the group formed by the fast-growing Rhizobium species. Both genomic species 1 and genomic species 2 contained a majority of strains which were capable of nodulating both $P$. vulgaris and Leucaena leucocephala, like $R$. tropici. However, they also contained strains with a nodulation phenotype restricted to $P$. vulgaris, like $\boldsymbol{R}$. leguminosarum bv. phaseoli and $R$. etli bv. phaseoli. Our data are the first evidence that in Europe species other than $R$. leguminosarum nodulate $P$. vulgaris.
\end{abstract}

The fast-growing bacteria that are able to induce nodules on the roots of leguminous plants are clustered in the genus Rhizobium. Each Rhizobium species is associated with a group of host plants $(5,14,19,23,34,36)$. The species Rhizobium leguminosarum is divided into the following three biovars: $R$. leguminosarum bv. viciae, which nodulates Vicia, Pisum, Lens, and Lathyrus species; R. leguminosarum bv. trifolii, which nodulates Trifolium species; and $R$. leguminosarum bv. phaseoli, which nodulates Phaseolus species (14). Plant specificity is encoded by genes located on a large plasmid, the symbiotic plasmid, which therefore, in $R$. leguminosarum, defines the biovar of its host bacteria.

The bacteria that are able to establish a nitrogen-fixing symbiosis with Phaseolus vulgaris are known to be both genotypically diverse and genetically distant $(6,27,30)$. In addition to $R$. leguminosarum, two other species that nodulate this plant have been characterized recently, Rhizobium etli and Rhizobium tropici $(23,36)$. $R$. etli includes the former $R$. leguminosarum bv. phaseoli type I American strains (21), which constitute one biovar, $R$. etli bv. phaseoli, and genetically related nonsymbiotic soil isolates from Mexico (35). Like $R$. leguminosarum bv. phaseoli, $R$. etli bv. phaseoli apparently nodulates only beans and possesses multiple copies of the nitrogenase reductase gene, nifH (22, $28,36)$. These plasmid-borne characteristics distinguish these organisms from other Rhizobium species. Partial sequences of 16S rRNA genes have been used to differentiate $R$. leguminosarum and $R$. etli (36). The third species, $R$. tropici, includes American strains (formerly $R$. leguminosarum bv. phaseoli type II strains) which are able to nodulate effectively both $P$. vulgaris and Leucaena spp. (23). These

\footnotetext{
* Corresponding author.

$\dagger$ Present address: Laboratoire de Flore Pathogène, Institut National de la Recherche Agronomique, F-21034 Dijon Cedex, France.
}

organisms have a single copy of nifH $(21,22)$. Two subgroups with distinctive phenotypic features (types IIA and IIB) have been identified in $R$. tropici (23).

All strains of $R$. etli and $R$. tropici originated in the Americas, most probably in Mexico. In Europe, the genetic structures of several Rhizobium populations isolated from beans have been studied $(10,17,24,41)$. The enzyme electrophoretic types of strains isolated from English fields were typical of $R$. leguminosarum and weakly polymorphic (41). In contrast, three taxa were delineated among 72 strains isolated from 20 different sites in French fields (10). One of these taxa was assigned to $R$. leguminosarum bv. phaseoli on the basis of a nodulation phenotype restricted to $P$. vulgaris associated with multiple copies of nifH, homology to an $R$. leguminosarum chromosomal DNA probe, and the results of a restriction fragment length polymorphism (RFLP) analysis. Within this taxon, the RFLP analysis revealed two main chromosomal groups, designated genomic groups A and B. Well-studied American strain CFN 42 also exhibited strong homology to the $R$. leguminosarum chromosomal probe and a restricted nodulation phenotype, but it had a distinct chromosomal type. These results correlated well with the fact that strain CFN 42 has recently been reclassified in the new species $R$. etli (36). Group A strains had RFLP patterns similar to those found in the predominant types of $R$ leguminosanum isolates obtained from root nodules of peas, lentils, and clover (17), but group B strains formed a group that was distinct from both typical strains of $R$. leguminosarum and CFN 42. Thus, the classification of group B strains as members of $R$. leguminosarum had to be verified. The two other taxa, designated genomic groups $\mathrm{D}$ and $\mathrm{E}$, were genomically distinct from the three previously recognized species that nodulate beans. Some of the strains belonging to groups $D$ and $E$ had the same nodulation phenotype as $R$. tropici since they were able to nodulate both $P$. vulgaris and Leucaena leucocephala. 
TABLE 1. Bacterial strains used in this study

\begin{tabular}{|c|c|c|c|c|}
\hline \multirow{2}{*}{ Strain } & \multicolumn{2}{|c|}{ Hybridization patterns ${ }^{a}$} & \multirow{2}{*}{$\begin{array}{l}\text { Host plant of original } \\
\text { culture }\end{array}$} & \multirow{2}{*}{ Source ${ }^{b}$} \\
\hline & pBA2-BamHI & pBA2-HindIII & & \\
\hline \multicolumn{5}{|l|}{ R. leguminosarum strains } \\
\hline ATCC $10004^{\mathrm{T}}$ & & & Pisum sativum & ATCC \\
\hline 8401(pRL1JI) & & & Phaseolus vulgaris & Young \\
\hline R. etli bv. phaseoli CFN $42^{\mathrm{T}}$ & & & Phaseolus vulgaris & Martinez-Romero \\
\hline \multicolumn{5}{|l|}{$R$. tropici strains } \\
\hline UMR $1899^{\mathrm{T}}\left(=\right.$ CIAT $\left.899^{\mathrm{T}}\right)($ type IIB) & & & Phaseolus vulgaris & Graham \\
\hline CFN 299 (type IIA) & & & Phaseolus vulgaris & Martinez-Romero \\
\hline R. meliloti RCR 2011 & & & Medicago sativa & RCR \\
\hline R. fredii USDA $205^{\mathrm{T}}$ & & & Glycine $\max$ & USDA \\
\hline R. loti NZP $2213^{\mathrm{T}}$ & & & Lotus tenuis & Jarvis \\
\hline $\begin{array}{l}\text { R. galegae HAMBI } 540^{\mathrm{T}}(=\mathrm{ATCC} \\
\left.43677^{\mathrm{T}}\right)\end{array}$ & & & Galega orientalis & Lindström \\
\hline \multicolumn{5}{|l|}{ Rhizobium spp. strains } \\
\hline $\mathrm{H} 441^{c}$ & d & c & Phaseolus vulgaris & MSDJ \\
\hline $\mathrm{R} 602 \mathrm{sp}^{c}$ & g & g & Phaseolus vulgaris & MSDJ \\
\hline $\mathrm{PhP} 222^{c}$ & g & h & Phaseolus vulgaris & MSDJ \\
\hline $\mathrm{PhD}^{c}{ }^{c}$ & g & i & Phaseolus vulgaris & MSDJ \\
\hline $\mathrm{H} 152^{c}$ & h & $\mathrm{m}$ & Phaseolus vulgaris & MSDJ \\
\hline $\operatorname{Ro}^{c} 4^{c}$ & $\mathbf{h}$ & $\mathbf{o}$ & Phaseolus vulgaris & MSDJ \\
\hline Ro70 & h & $\mathbf{o}$ & Phaseolus vulgaris & MSDJ \\
\hline $\operatorname{Ro68}^{c}$ & h & 1 & Phaseolus vulgaris & MSDJ \\
\hline $\operatorname{Ro} 24^{c}$ & h & $\mathbf{p}$ & Phaseolus vulgaris & MSDJ \\
\hline BR 864 & & & Leucaena leucocephala & Martinez-Romero \\
\hline ORS 1007 & & & Acacia laeta & Dreyfus \\
\hline UPMCA 36 & & & Cicer arietinum & Fernandez \\
\hline 522 & & & Cicer arietinum & Fernandez \\
\hline
\end{tabular}

${ }^{a}$ The French isolates obtained from Phaseolus beans were previously characterized by hybridizing endonuclease-restricted DNAs with a 16S rDNA probe, pBA2 (10). Each of the three taxa identified by the RFLP analysis is represented by strains having one of the pBA2-BamHI hybridization patterns (pattern d, $g$, or h). The pBA2-HindIII patterns characterized variants within groups.

${ }^{b}$ Sources: ATCC, American Type Culture Collection, Rockville, Md.; Young, J. P. W. Young, John Innes Institute, Norwich, United Kingdom; Martinez-Romero, E. Martinez-Romero, Centro de Investigacion sobre Fijacion de Nitrogeno, Universidad Nacional Autonoma de Mexico, Cuernavaca, Mexico; Graham, P. Graham, Department of Soil Science, University of Minnesota, St. Paul; RCR, Rothamsted Collection of Rhizobium, Harpenden, Hertforshire, United Kingdom; USDA, Rhizobium Culture Collection, Beltsville Agricultural Research Center, Beltsville, Md.; Jarvis, B. D. W. Jarvis, Department of Microbiology and Genetics, Massey University, Palmerson North, New Zealand; Lindström, K. Lindström, Department of Microbiology, University of Helsinki, Helsinki, Finland; MSDJ, Institut National de la Recherche Agronomique (INRA), Microbiologie des Sols, Dijon, France; Dreyfus, B. Dreyfus, O.R.S.T.O.M., Dakar, Senegal; Fernandez, M. P. Fernandez, Laboratoire d'Ecologie Microbienne, Université Claude Bernard Lyon I, Villeurbanne, France.

${ }^{c}$ French isolates obtained from Phaseolus beans.

Broad-host-range strains in group D possessed one copy of nifH, like $R$. tropici, whereas broad-host-range strains in group $\mathrm{E}$ did not exhibit homology to nifH. Group E contained about one-third of the bean rhizobia obtained from two natural French field populations $(10,17,24)$.

In this study, DNA-DNA hybridization and analysis of partial 16S ribosomal DNA (rDNA) sequences were used to examine the taxonomic status of French Rhizobium strains that nodulate Phaseolus beans and represent the three groups defined previously (groups B, D, and E) $(10,17)$.

\section{MATERIALS AND METHODS}

Bacterial strains. The bacterial strains used in this study are listed in Table 1 . Cells were grown at $28^{\circ} \mathrm{C}$ in TY medium (1).

DNA extraction. DNA was extracted and purified by using the procedure of Brenner et al. (2).

DNA-DNA hybridization. Native DNA was labeled by nick translation (29) with tritium-labeled nucleotides (Amersham International, Amersham, England). Levels of DNA relatedness were determined by using the S1 nuclease-trichloroacetic method (12). DNA-DNA hybridization tests were carried out at $70^{\circ} \mathrm{C}$ by using labeled DNAs from strains
ATCC $10004^{\mathrm{T}}(\mathrm{T}=$ type strain $)$, CIAT $899^{\mathrm{T}}, \mathrm{R} 602 \mathrm{sp}$, and $\mathrm{H} 152$ as probes.

Nucleotide sequence of $16 \mathrm{~S}$ rRNA genes. Total DNA was amplified by using primers FGPS6-63 (5'-GGAGAGTT $\underline{A}$ GATCTTGGCTC-3') and FGPS659'-64 (5'-CACCGCTA CACCAGGAATTC-3') and a standard protocol (26), which yielded DNA bands of the expected size $(650 \mathrm{bp})$ (data not shown); the amplified fragments were then digested with $B g l$ II and $E c o$ RI, which have sites in the primers (underlined regions), cloned into BamHI-EcoRI-cut pBluescript II SKvector (Stratagene, La Jolla, Calif.), and transformed into Escherichia coli $\mathrm{DH} 5 \alpha \mathrm{F}^{\prime}$ (Bethesda Research Laboratories) as described by Maniatis et al. (20). Ten white clones (i.e., clones with insertions) were pooled and isolated from Quiagen midi columns (Diagen, Hilden, Germany). These doublestranded fragments were sequenced in both directions as described by Sanger et al. (33), using primers FGPS6-63 (see above) and FGPS 305'-78 (5'-CCAGTGTGGCCGGTCGC CCTCTC-3') and a T7 sequencing kit (Pharmacia, St. Quentin-Yvelynes, France).

Alignment of sequences and cluster analysis. Nucleotide sequences were aligned by using the Clustal program (13) and were visualized by using the MASE software (9) and a Sun workstation, and the neighbor-joining algorithm (32) was used to determine the phylogenetic relationships from 
TABLE 2. Levels of DNA relatedness between strains of Rhizobium species

\begin{tabular}{|c|c|c|c|c|}
\hline \multirow{2}{*}{ Strain } & \multicolumn{4}{|c|}{$\%$ Reassociation at $70^{\circ} \mathrm{C}$ with labeled DNA from: } \\
\hline & ATCC $10004^{\mathrm{T}}$ & CIAT $899^{\mathrm{T}}$ & R602sp & H152 \\
\hline \multicolumn{5}{|l|}{ R. leguminosarum strains } \\
\hline ATCC $10004^{\mathrm{T}}$ & 100 & & 21 & 16 \\
\hline 8401(pRL1J1) & 100 & & & \\
\hline $\mathbf{H} 441^{a}$ & 100 & & & \\
\hline R. etli CFN $42^{\mathrm{T}}$ & 45 & & 18 & 14 \\
\hline \multicolumn{5}{|l|}{ R. tropici strains } \\
\hline CIAT $899^{\mathrm{T}}$ (type IIB) & 15 & 100 & 13 & 10 \\
\hline CFN 299 (type IIA) & 19 & 36 & 15 & 13 \\
\hline R. meliloti RCR 2011 & & & 7 & 15 \\
\hline R. fredii USDA $205^{\mathrm{T}}$ & & & 10 & 17 \\
\hline R. loti NZP $2213^{\mathrm{T}}$ & & & 5 & 6 \\
\hline R. galegae HAMBI $540^{\mathrm{T}}$ & & & 11 & 11 \\
\hline \multicolumn{5}{|l|}{ Rhizobium spp. strains } \\
\hline BR 864 & 23 & & 11 & 10 \\
\hline ORS 1007 & & & 7 & 16 \\
\hline UPMCA 36 & & & 7 & 6 \\
\hline 522 & & & 3 & 7 \\
\hline \multicolumn{5}{|l|}{ Genomic species 1 strains } \\
\hline $\mathrm{R} 602 \mathrm{sp}^{a}$ & 23 & 19 & 100 & 18 \\
\hline $\mathrm{PhP} 222^{a}$ & 23 & & 91 & 11 \\
\hline $\mathrm{PhD} 12^{a}$ & & & 76 & 14 \\
\hline \multicolumn{5}{|l|}{ Genomic species 2 strains } \\
\hline $\mathrm{H} 152^{a}$ & 10 & 12 & 10 & 100 \\
\hline $\operatorname{Ro} 84^{a}$ & 13 & & 8 & 88 \\
\hline $\operatorname{Ro} 70^{a}$ & 11 & & 11 & 93 \\
\hline $\operatorname{Ro6}^{a}$ & & & 13 & 74 \\
\hline $\operatorname{Ro} 24^{a}$ & & & 11 & 95 \\
\hline
\end{tabular}

${ }^{a}$ French isolates obtained from Phaseolus beans.

Jukes-Cantor distances (15). Gap-containing positions were omitted from computations.

Nucleotide sequence accession numbers. For comparison we used the sequences of Rhizobium sp. strain FL27 (Phaseolus spp.) (GenBank accession number M55234), Rhizobium loti (GenBank accession number X63825), Rhizobium galegae (GenBank accession number X63823), Rhizobium sp. strain TAL 1145 (Leucaena spp.) (GenBank accession number X63824), $R$. tropici type IIB strain CIAT $899^{\mathrm{T}}$ (GenBank accession number M55233), $R$. tropici type IIA strain CFN 299 (EMBL accession number X67234), $R$. etli Olivia 4 (GenBank accession number M55235), Rhizobium sp. strain Or191 (Medicago spp.) (GenBank accession number M55236), $R$. leguminosarum bv. phaseoli 8002 (GenBank accession number M55494), Rhizobium meliloti ATCC $9930^{\mathrm{T}}$ (GenBank accession number M55242), R. meliloti CC169 (GenBank accession number M55243), Rhizobium fredii (GenBank accession number M74163), Agrobacterium tumefaciens ICPB TT111 (EMBL accession number X67223), Agrobacterium rhizogenes ATCC 15834 (EMBL accession number X67224), Agrobacterium rubi ATCC 13335 (EMBL accession number X67228), Agrobacterium vitis NCPPPB 3554 (EMBL accession number X67225), Bradyrhizobium japonicum USDA 110 (GenBank accession number M55485), Azorhizobium caulinodans (GenBank accession number M55491), Rhodopseudomonas palustris (GenBank accession number M55496), Rhodospirillum rubrum (GenBank accession number M55497), and Rhodobacter sphaeroides (GenBank accession number M55498). The partial 16S rDNA sequence of Rhizobium sp. strain $\mathrm{H} 152$ determined in this study was deposited in the GenBank data library under accession number L19662.

\section{RESULTS}

DNA-DNA hybridizations. The percentages of relative DNA homology at $70^{\circ} \mathrm{C}$ with reference DNAs from $R$. leguminosarum ATCC $10004^{\mathrm{T}}$, $R$. tropici CIAT $899^{\mathrm{T}}$, and strains R602sp and $\mathrm{H} 152$ are shown in Table 2 for 22 strains.

Of the nine French strains examined, only one, H441, was $100 \%$ related to type strain ATCC 10004 of $R$. leguminosarum. The eight other strains fell into two DNA relatedness groups (genomic species). Genomic species 1 contained three strains which were 76 to $91 \%$ related to strain R602sp. The five other strains, which were 73 to $95 \%$ related to strain $\mathrm{H} 152$, formed genomic species 2. The genomic species 2 strains exhibited low relative binding ratios (less than $18 \%$ ) with genomic species 1 strains. Strains belonging to both genomic species were less than $21 \%$ related to reference strains belonging to other Rhizobium species, including $R$. leguminosarum, $R$. etli, $R$. tropici type IIA and IIB, $R$. loti, $R$. galegae, $R$. meliloti, $R$. fredii, and unclassified field rhizobia isolated from L. leucocephala, Acacia laeta, and Cicer arietinum.

It should be noted that $R$. tropici type IIA strain CFN 299 was only $36 \%$ related to $R$. tropici type IIB strain CIAT $899^{\mathrm{T}}$, a value similar to the value found previously by Martinez-Romero et al. (23). $R$. etli CFN $42^{\mathrm{T}}$ was found to be $45 \%$ related to type strain ATCC $10004^{\mathrm{T}}$ of $R$. leguminosarum, which also confirmed the relative levels of DNA-DNA hybridization previously obtained between CFN $42^{\mathrm{T}}$ and two $R$. leguminosarum strains (35).

Nucleotide sequence analysis of $16 S$ rDNA. Partial $16 \mathrm{~S}$ rDNA sequences containing 246 nucleotides were obtained for genomic species 1 strain R602sp and genomic species 2 
R. Sp. H152

R. sp. R602sp

R. loti

$R$. galegae

$\mathrm{R}$. Leucaena

R. tropici CIAT899

R. tropici IIA

R. etli Olivia

R. sp. medicago 191

R. leguminosa rum8002

R. meliloti ATCC 9930

$R$, meliloti CC169

$R$. fredii

A. tumefaciens

A. rhizogenes

A. rubi

A.vitis

B. Japonicum USDA110

Azorhizobium

Rhodopseudomonas

Rhodobacter

Rhodospirillum
1
11
21
31
41

AGGCTTAACA CATGCAAGTC GAGCG--CCC CGCAAGGGG- -AGCGGCAGA AGGCTTAACA CATGCAAGTC GAGCG--CCC CGCAAGGGG- -AGCGGCAGA AGGCTTAACA CATGCAAGTC GAGCG--CCT CGCAAGAGG- -AGCGGCAGA AGGCTTAACA CATGCAAGTC GAGCG--CCT CGCAAGAGG- -AGCGGCAGA AGGCTTAACA CATGCAAGTC GAGCG--CCC CGCAAGGGG- -AGCGGCAGA AGGCTTAACA CATGCAAGTC GAGCG--CCC CGCAAGGGG- -AGCGGCAGA AGGCTTAACA CATGCAAGTC GAGCG--CCC CTCGAGGGG- -AGCGGCAGA AGGCTTAACA CATGCAAGTC GAGCG--CCC CGCAAGGGG- -AGCGGCAGA AGGCTTAACA CATGCAAGTC GAGCG--CCC CGCAAGGGG- -AGCGGCAGA AGGCTTAACA CATGCAAGTC GAGCG--CCC CGCAAGGGG- -AGCGGCAGA AGGCTTAACA CATGCAAGTC GAGCG--CCC CGCAAGGGG- -AGCGGCAGA AGGCTTAACA CATGCAAGTC GAGCG--CCC CGCAAGGGG- -AGCGGCAGA AGGCTTAACA CATGCAAGTC GAGCG--CCC CGCAAGGGG- -AGCGGCAGA AGGCTTAACA CATGCAAGTC GAACG--CCC CGCAAGGGG- -AGTGGCAGA AGGCTTAACA CATGCAAGTC GAGCG--CCC CGCAAGGGG- -AGCGGCAGA AGGCTTAACA CATGCAAGTC GAACG--CTC CGCAAGGAG- -AGTGGCAGA AGGCTTAACA CATGCAAGTC GAGCG--CCT CGCAAGAGG- -AGCGGCAGA AGGCTTAACA CATGCAAGTC GAGCGGGCGT AGCAATACGT CAGCGGCAGA AGGCTTAACA CATGCAAGTC GAACGGGCCC -TTCG-GG-T CAGTGGCAGA AGGCTTAACA CATGCAAGTC GAACGGGCGT AGCAATACGT CAGTGGCAGA AGGCTTAACA CATGCAAGTC GAGCGC-CGT AGCAATACGG -AGCGGCAGA AGGCTTAACA CATGCAAGTC GAACGCATCC CTTCG-GGAT GAGTGGCGCA
51
61
71
81
91

CGGGTGAGTA ACGCGTGGGA ATCTACCCAT CTCTACGGAA TAACTCAGGG CGGGTGAGTA ACGCGTGGGA ACGTACCCTT TACTACGGAA TAACGCAGGG CGGGTGAGTA ACGCGTGGGA ATCTACCCAT CTCTACGGAA CAACTCCGGG CGGGTGAGTA ACGCGTGGGA ATCTACCCAT CCCTACGGAA CAACTCCGGG CGGGTGAGTA ACGCGTGGGA ATCTACCCTT TTCTACGGAA TAACGCAGGG CGGGTGAGTA ACGCGTGGGA-ATCTACCTTT TGCTACGGAA TAACGCAGGG CGGGTGAGTA ACGCGTGGGA ATCTACCCTT TTCTACGGAA TAACGCAGGG CGGGTGAGTA ACGCGTGGGA ACGTACCCTT TACTACGGAA TAACGCAGGG

CGGGTGAGTA ACGCGTGGGA ACGTACCCTT TACTACGGAA TAACGCATGG

R. sp. R602sp

R. loti

$R$. galegae

$R$. Leucaena

R. tropici CIAT899

R. tropici IIA

R. etli Olivia

R. sp. medicago 191

$R$. leguminosarum8002

R. meliloti ATCC9930 CGGGTGAGTA ACGCGTGGGA ATCTACCCTT TTCTACGGAA TAACGCAGGG

R. meliloti CC169

R. fredii

A. tumefaciens

A. Ihizogenes

A. rubi

A. vitis CGGGTGAGTA ACGCGTGGGA ATCTACCCTT TTCTACGGAA TAACGCAGGG CGGGTGAGTA ACGCGTGGGA ATCTACCCTT TTCTACGGAA TAACGCAGGG CGGGTGAGTA ACGCGTGGGA ATCTACCGTG CCCTGCGGAA TAGCTCCGGG CGGGTGAGTA ACGCGTGGGA ATCTACCCTT TTCTACGGAA TAACGCAGGG CGGGTGAGTA ACGCGTGGGA ATCTACCCAA CCCTGCGGAA TAGCTCTGGG CGGGTGAGTA ACGCGTGGGA ATCTACCGTA CCCTACGGAA TAGCTCCGGG

B. japonicum USDA110 CGGGTGAGTA ACGCGTGGGA ACGTACCTTT TGGTTCGGAA CAACACAGGG Azorhizobium Rhodopseudomonas Rhodobacter Rhodospirillum CGGGTGAGTA ACGCGTGGGA ACGTGCCCTT CAGTTCGGAA TAACCCAGGG CGGGTGAGTA ACGCGTGGGA ACGTACCTTT TGGTTCGGAA CAACACAGGG CGGGTGAGTA ACGCGTGGGA ATGTGCCCAA AGGTACGGAA CAACCAAGGG CGGGTGAGTA ACACGTGGGA ACGTACCTTG GAGTGCGGAA TAATCTTTGG

R. sp. H152

R. sp. R602sp

R. Loti

$R$. ga legae

$R$. Leucaena

R. tropici CIAT899

R. tropici IIA

R. etli Olivia

R. sp. medicago 191

101

111

121

131

141

AAACTTGTGC TAATACCGTA TACGCCCTTC GGGGGAAAGA TTTATCGGAG AAACTTGTGC TAATACCGTA TGTGCCCTTC GGGGGAAAGA TTTATCGGTA AAACTGGAGC TAATACCGTA TACGTCCTTC GGGAGAAAGA TTTATCGGAG AAACTGGAGC TAATACCGTA TACGCCCTTC GGGGGAAAGA TTTATCGGGG AAACTTGTGC TAATACCGTA TGTGTCCTTC GGGAGAAAGA TTTATCGGGG AAACTTGTGC TAATACCGTA TGTGTCCTTC GGGAGAAAGA TTTATCGGCA AAACTTGTGC TAATACCGTA TGTGTCCTTC GGGAGAAAGA TTTATCGGGA AAACTTGTGC TAATACCGTA TGTGCCCTTT GGGGGAAAGA TTTATCGGTA AAACGTGTGC TAATACCGTA TGTGCCCTTT GGGGGAAAGA TTTATCGGTA R. leguminosarum8002 AAACTTGTGC TAATACCGTA TGTGTCCTTC GGGAGAAAGA TTTATCGGTC R. meliloti ATCC9930 AAACTTGTGC TAATACCGTA TGAGCCCTTC GGGGGAAAGA TTTATCGGGA R. meliloti CC169 AAACTTGTGC TAATACCGTA TAAGCCCTTC GGGGGAAAGA TTTATCGGGA R. fredii

A. tumefaciens

A. rhizogenes

A. rubi

A. vitis AAACTTGTGC TAATACCGTA TGAGCCCTTC GGGGGAAAGA TTTATCGGGA AAACTGGAAT TAATACCGCA TACGCCCTAC GGGGGAAAGA TTTATCGGGG AAACTTGTGC TAATACCGTA TGTGTCCTTC GGGAGAAAGA TTTATCGGGA AAACTGGAAT TAATACCGCA TACGCCCTAC GGGGGAAAGA TTTATCGgGG AAACTGGAAT TAATACCGTA TACGCCCTTC GGGGGAAAGA TTTATCGGGG B. japonicum USDA110 AAACTTGTGC TAATACCGGA TAAGCCCTTA CGGGGAAAGA TTTATCGCCG Azorhizobium Rhodopseudomonas Rhodobacter Rhodospirillum AAACTTGGGC TAATACCGGA TACGTCCGAA AGGAGAAAGA TTGATCGCTG AAACTTGTGC TAATACCGGA TAAGCCCTTA CGGGGAAAGA TTTATCGCCG AAACTTTGGC TAATACCGTA TGTGCCCGAG AGGGGAAAGA TTTATTGCCT AAACGAGGAC TAATACCGCA TACGCCCTTA GgGGGAAAGA TTTATCGCTC 


\begin{tabular}{|c|c|c|c|c|c|}
\hline & 151 & 161 & 171 & 181 & 191 \\
\hline R.sp. H152 & ATGGATGAGC & CCGCGTTGGA & TTAGCTAGTT & GGTGGGGTAA & AGGCCTACCA \\
\hline R. sp. $R 602 s p$ & AGGGATCGGC & CCGCGTTGGA & TTAGCTAGTT & GGTGGGGTAA & AGGCCTACCA \\
\hline R. loti & ATGGATGAGC & CCGCGTTGGA & TTAGCTAGTT & GGTGGGGTAA & TGGCCTACCA \\
\hline R. galegae & ATGGATGAGC & CĊGCGTTGGA & TTAGCTAGTT & GGTGGGGTAA & AGGCCTACCA \\
\hline R. Leucaena & AAGGATGAGC & CCGCGTTGGA & TTAGCTAGTT & GGTGGGGTAA & AGGCCTACCA \\
\hline R,tropici CIAT899 & AGAGATGAGC & CCGCGTTGGA & TTAGCTAGTT & GGTGGGGTAA & AGGCCTACCA \\
\hline R, tropici IIA & AAGGATGAGC & CCGCGTTGGA & TTAGCT & GGTGGC & AGGCCI \\
\hline R, etli Olivia & ATCGGC & CCGCGTTGGA & TTAGCTAGTT & GGTGGG & \\
\hline R.sp. medicago 191 & AAGGATCGGC & CCGCGTTGGA & TTAGCTAGTT & GGTGGGGTAA & AGGCCTACCA \\
\hline R. leguminosarum8002 & AAGGATGAGC & CCGCGTTGGA & TTAGCTAGTT & GGTGGGGTAA & AGGCCTACCA \\
\hline R. meliloti ATCC 9930 & AAGGATGAGC & CCGCGTTGGA & TTAGCTAGTT & GGTGGGGTAA & AGGCCTACCA \\
\hline R. meliloti CC169 & AAGGATGAGC & CCGCGTTGGA & TTAGCTAGTT & GGTGG & AGGCCT \\
\hline R.fredii & AAG & CCGCGTTGGA & TTAGCT & GGTGG & AGGCCT \\
\hline A. tumefaciens & TATGATGAGC & CCGCGTTGGA & TTAGCT & GGTGG & AGGCCT \\
\hline ogenes & AAGGATGAGC & CCGCGTTGGA & TTAGCTAGTT & GGTGG & AGGCCT \\
\hline A. 2 & ATGGAI & CCGCGTTGGA & TTAGCTAGTT & GGTGG & AGGCCT \\
\hline A. $v$ & GC & CCGCGTTGGA & TTAGCTAGTT & GGTGG & AGGCCT \\
\hline cum USDA110 & AAA & CCGCGTCTGA & TTAGCT & GGTAC & CGGCCT \\
\hline $\mathrm{AzO}$ & AAG & CCGCGTCTGA & TT & GGTG & $\mathrm{CA}$ \\
\hline domonas & AAAG & CCGCGTCTGA & TT & GGTG & $\mathrm{CA}$ \\
\hline Rhod & TTGGAGC & CCGCGTCTGA & TTAGCT & GGTGC & CGGCCC \\
\hline \multirow[t]{2}{*}{ Rhodospirillum } & CAAGATCGGC & CCGCGTCCGA & TAGCT & GGCGG & TGGCCC \\
\hline & 20 & & & 231 & \\
\hline & AGGCG & CCATAGCTGG & TTGAG & TGATC & CATTGG \\
\hline 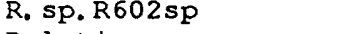 & AT & CCATAGCTGG & TCTGAC & $\mathrm{ECA}$ & ET \\
\hline R. $J$ & AGG & CCATAGCTGG & TCTGAG & TGATC & CATTGGGACT \\
\hline R. galegae & ACGAT & CCATAGCTGG & CTGAG & TGATCZ & CATTGGGACT \\
\hline R. Leucaena & AGG & CCATAGCTGG & CCTGAG & TGATC & CATTGG \\
\hline R.tropici CIAT899 & AGGCC & CCATAGCTGG & TGAGAGGA & TGATCAGCCA & CATTGG \\
\hline R. tropici & $\mathrm{T}$ & AGCTGG & TGP & TGATC & CATTC \\
\hline La & & ;G & & A & $\mathrm{ET}$ \\
\hline medi & & & & & \\
\hline R. leguminosarum 8002 & AGGCGACGAT & CCATAGCTGG & ICTGAGAGGA & TGATC & CATTGG \\
\hline R. meliloti ATCC9930 & AGGCGACGAT & CCATAGCTGG & CTGAGAGGA & TGATCAGCCA & CATTGGGACT \\
\hline R.meliloti CC169 & AGGCGACGAT & CCATAGCTGG & CCTGAGAGGA & TGATC & CATTGGGACT \\
\hline R.fredii & AGGCG & CCATAGCTGG & ICTGAG & TGATC & CATTGG \\
\hline iens & AC & ;G & A & TGAT & TTC \\
\hline A. I & $\mathrm{T}$ & $\mathbf{G}$ & A & A & $\mathbf{T}$ \\
\hline & $\mathrm{T}$ & & & AT & ACT \\
\hline A. $v$ & AGGCGACGAT & CCATAGCTGG & [CTGAG & TGATC & CATTGGGACT \\
\hline B. japon & AGGCGACGAT & CAGTAGCTGG & TCTGAG & TGATCAGCCA & CATTGGGACT \\
\hline Azorhizobium & AGGCGACGAT & AGTAGCTGG & CTGAGAGGA & TGATCAGCCA & CATTGGGACT \\
\hline hodopseudomor & AGGCGACGAT & GTAGCTGG & TGAGAGGA & TGATCAGCCA & CATTGGGACT \\
\hline & & & & $\mathrm{CA}$ & $\Gamma \mathrm{T}$ \\
\hline Rhodospiri & AT & & & AAC & GACT \\
\hline
\end{tabular}

FIG. 1. 16S rDNA sequences of Rhizobium sp. strain H152 (this study), Rhizobium sp. strain R602sp (identical to Rhizobium sp. strain FL27) (this study), $R$. loti, $R$. galegae, Rhizobium sp. strain Tal 1145 (R. Leucaena), $R$. tropici CIAT $899^{\mathrm{T}}$ (type IIB), $R$. tropici CFN 299 (type IIA) (R. tropici IIA), $R$. etli Olivia 4, Rhizobium sp. strain Or191 (R. sp. medicago 191), $R$. leguminosarum bv. phaseoli 8002, $R$. meliloti ATCC $9930^{\mathrm{T}}, R$. meliloti CC169, R. fredii USDA $205^{\mathrm{T}}$, Agrobacterium tumefaciens ICPB TT111, Agrobacterium rhizogenes ATCC 15834, Agrobacterium rubi ATCC 13335, Agrobacterium vitis NCPPPB 3554, B. japonicum USDA 110, Azorhizobium caulinodans, Rhodopseudomonas palustris, Rhodospirillum rubrum, and Rhodobacter sphaeroides. The underlined sequence TCGA at coordinates 32 through 35 represents the site of the 72-nucleotide insertion unique to $R$. tropici type IIA strain CFN 299 (TCCTT CAAGCAAGCT TGAAGGATT TACTCCTTGGA AAAGAAGATC AAGAGCGCTT CTAAGAAGCT TTCTTGATGGA) (39), which was deleted for clarity.

strain H152. These sequences were within the region from position 20 to position 338 (E. coli numbering system [4]), which was previously used to determine phylogenetic relationships among rhizobia $(8,36,42)$. The sequences were compared with those found for other Rhizobium and Bradyrhizobium species and some other genera belonging to the alpha subdivision (40) of the Proteobacteria (37) (Fig. 1). The sequences of strains R602sp and H152 were included in the group containing fast-growing Rhizobium species (Fig. 2 ). The sequence of strain R602sp was identical to that of Rhizobium sp. strain FL27, an unclassified strain isolated from Phaseolus beans (27). These two strains formed a lineage independent from the lineages of other Rhizobium strains and more closely related to $R$. etli than to other Rhizobium species. Genomic species 2 strain $\mathrm{H} 152$ also formed an independent lineage; the most closely related organisms to this lineage were $R$. galegae and $R$. lot $i$ but no other strains isolated from beans (Fig. 2).

\section{DISCUSSION}

Of the nine strains examined in this study for their taxonomic status, strain $\mathrm{H} 441$ was a member of the taxon which we previously hypothesized as belonging to the spe- 


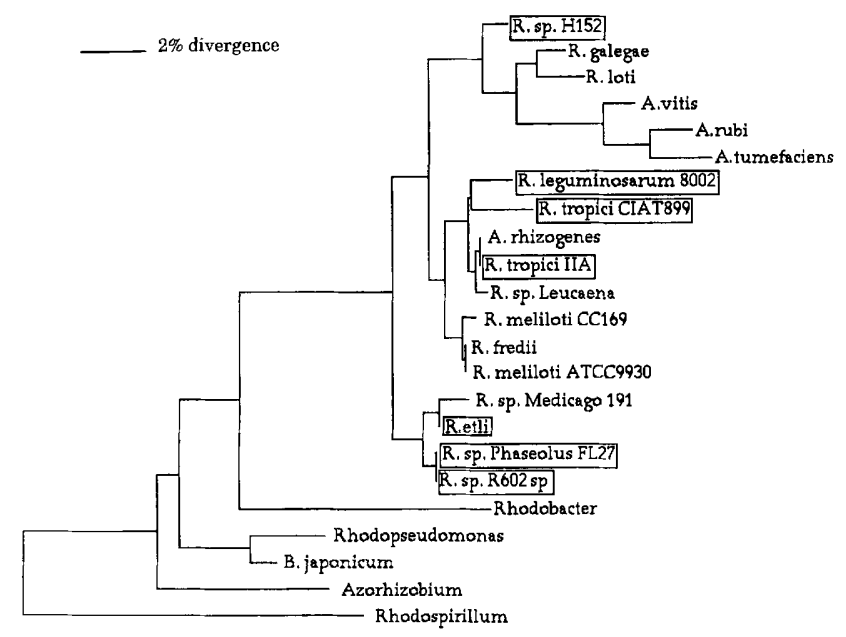

FIG. 2. Phylogenetic tree showing relationships among Phaseolus bean rhizobia and other strains of Rhizobium spp., Agrobacterium spp., and related bacteria based on partial 16S rDNA sequences. The tree was derived from a Jukes-Cantor (15) distance matrix by using the neighbor-joining method (32) and the sequences shown in Fig. 1. The taxa in boxes are the taxa that infect $P$. vulgaris. Abbreviations: R.sp. H152, Rhizobium sp. strain H152; R. tropici IIA, $R$. tropici type IIA strain CFN 299; R. sp. Leucaena, Rhizobium sp. strain Tal 1145; R. sp. Medicago 191, Rhizobium sp. strain Or191; R. sp. Phaseolus FL27, Rhizobium sp. strain FL27; R. sp. R602sp, Rhizobium sp. strain R602sp.

cies $R$. leguminosarum $(10,17)$. Within this taxon, two main groups (groups A and B) of closely related genotypes were found by an RFLP analysis. In this study, we verified that H441 and closely related strains (group B) belonged to the species $R$. leguminosarum since strain H441 was $100 \%$ related to ATCC 10004, the type strain of this species. Members of the other group (group A) were not included in this study because their chromosomal types were similar to the predominant types of $R$. leguminosarum isolates obtained from root nodules of peas, lentils, and clover (17) and to the type strain of $R$. leguminosarum (16).

The eight other strains examined in this study that belonged to the other RFLP groups defined previously (10) were distributed in two new genomic Rhizobium species as revealed by DNA-DNA hybridization and $16 \mathrm{~S}$ rDNA sequence analysis data. The DNA relatedness data confirmed the previous groupings since strains belonging to the same RFLP group belonged to the same genomic species. This is the first evidence that in Europe species other than $R$. leguminosanum are capable of nodulating Phaseolus beans in the field. However, no strains related to $R$. etli and $R$. tropici have been identified in the sample of French isolates obtained from Phaseolus beans. So far, members of $R$. etli and $R$. tropici have been found only in the Americas.

Most strains of the two new genomic species identified in this study were capable of nodulating both $P$. vulgaris and $L$. leucocephala (10), a characteristic that they had in common with $R$. tropici. However, the phylogenetic analysis indicated that despite its broad host range, genomic species 1 strain R602sp is more closely related to $R$. etli than to $R$. tropici. Conversely, $R$. leguminosarum is more closely related to $R$. tropici than to $R$. etli. However, it has been suggested that reliable conclusions cannot be drawn about relationships below the genus level by using the short segment of $16 \mathrm{~S}$ rDNA that was sequenced (42). Neverthe- less, some strains belonging to both genomic species 1 and 2 had a nodulation phenotype restricted to $P$. vulgaris associated with the presence of three copies of the nifH gene (such as strains PhD12 and Ro84 used in this study), which is characteristic of the pSym of $R$. leguminosanum bv. phaseoli and $R$. etli bv. phaseoli. It was suggested previously that in such strains, the Phaseolus restricted phenotype was acquired by horizontal transfer of a pSym from an $R$. leguminosarum bv. phaseoli strain (10). Until now, the recognized Rhizobium species that nodulate beans have been clearly associated with specific symbiotic characteristics, in particular the range of hosts that they are able to nodulate. Thus, host range has been considered a helpful diagnostic test to identify such bacteria. The fact that there may be exchanges of symbiotic characteristics between species in the field will hamper the identification of bean isolates.

Since DNA-DNA hybridization is considered the standard arbiter for the designation of species $(25,38)$, our finding of two new genomic species should lead to the creation of new Rhizobium species. The phenotypic characterization of these organisms is currently in progress in our laboratory. $P$. vulgaris is known to be a promiscuous host for Rhizobium species, in contrast to other legumes grown in temperate areas. This plant can be nodulated under laboratory conditions by different strains of Rhizobium spp. and even by Bradyrhizobium spp. isolated from other genera of legumes $(3,6,7,11,18,22,31)$ and in the field by $R$. leguminosarum strains harboring typical $R$. leguminosarum bv. viciae and $R$. leguminosarum bv. trifolii symbiotic plasmids (17). The identification of two additional genomic species of rhizobia able to nodulate $P$. vulgaris further demonstrates the low level of specificity of the Phaseolus-Rhizobium symbiosis and helps to explain some of the genetic diversity found in Rhizobium species that nodulate beans $(6,27,30)$.

Despite the recent creation of two novel species, $R$. etli and $R$. tropici, and the possible creation of two other species, we can expect larger taxonomic diversity among Rhizobium strains isolated from Phaseolus beans. The two subgroups of the species $R$. tropici were found to be only $36 \%$ related (23), and $R$. etli contains genetically distinct strains (27) which were grouped in a single species mainly on the basis of similar sequences in a partial region of the $16 \mathrm{~S}$ rDNA (36). Moreover, until now, studies of the bean symbionts have been limited to the Americas and a part of occidental Europe. As further isolations are made from other geographic areas, additional new species may be created to reflect, at a taxonomic level, the great diversity of bacteria capable of nodulating beans.

\section{ACKNOWLEDGMENTS}

We thank B. Dreyfus, P. Graham, B. D. W. Jarvis, K. Linström, E. Martinez-Romero, and J. P. W. Young for giving us reference strains, E. Martinez-Romero for giving us a preprint of a manuscript, and F. Revoy and C. Chapelon for technical assistance.

\section{REFERENCES}

1. Beringer, J. E. 1974. R factor transfer in Rhizobium leguminosarum. J. Gen. Microbiol. 84:188-198.

2. Brenner, D. J., A. C. McWorter, J. K. Leete Knutson, and A. G. Steigerwalt. 1982. Escherichia vulneris: a new species of Enterobacteriaceae associated with human wounds. J. Clin. Microbiol. 15:1133-1140.

3. Bromfield, E. S. P., and L. R. Barran. 1990. Promiscuous nodulation of Phaseolus vulgaris, Macroptilium atropurpureum, and Leucaena leucocephala by indigenous Rhizobium meliloti. Can. J. Microbiol. 36:369-372.

4. Brosius, J., T. J. Dull, D. D. Sleeter, and H. F. Noller. 1981. 
Gene organization and primary structure of a ribosomal RNA operon from E. coli. J. Mol. Biol. 148:107-127.

5. Chen, W. X., G. S. Li, Y. L. Qi, E. T. Wang, H. L. Yuan, and J. L. Li. 1991. Rhizobium huakuii sp. nov. isolated from the root nodules of Astragalus sinicus. Int. J. Syst. Bacteriol. 41:275280.

6. Crow, V. L., B. D. W. Jarvis, and R. M. Greenwood. 1981. Deoxyribonucleic acid homologies among acid-producing strains of Rhizobium. Int. J. Syst. Bacteriol. 31:152-172.

7. Eardly, B. D., D. B. Hannaway, and P. J. Bottomley. 1985. Characterization of rhizobia from ineffective alfafa nodules: ability to nodulate bean plants [Phaseolus vulgaris (L.) Savi.]. Appl. Environ. Microbiol. 50:1422-1427.

8. Eardly, B. D., J. P. W. Young, and R. K. Selander. 1992. Phylogenetic position of Rhizobium sp. strain Or 191, a symbiont of both Medicago sativa and Phaseolus vulgaris, based on partial sequences of the 16S rRNA and nifH genes. Appl. Environ. Microbiol. 58:1809-1815.

9. Faulkner, D. A., and J. Jurka. 1988. Multiple aligned sequence editor (MASE). Trends Biochem. Sci. 13:321-322.

10. Geniaux, E., G. Laguerre, and N. Amarger. Mol. Ecol., in press.

11. Graham, P. H., and C. A. Parker. 1964. Diagnostic features in the characterization of the root-nodule bacteria of legumes. Plant Soil 20:383-386.

12. Grimont, P. A. D., M. Y. Popoff, F. Grimont, C. Coynault, and M. Lemelin. 1980. Reproducibility and correction study of three deoxyribonucleic acid hybridization procedures. Curr. Microbiol. 4:325-330.

13. Higgins, D. G., and P. M. Sharp. 1988. Clustal: a package for performing multiple alignment on a microcomputer. Gene 73: $237-244$.

14. Jordan, D. C. 1984. Family III. Rhizobiaceae, p. 234-242. In N. R. Krieg and J. G. Holt (ed.), Bergey's manual of systematic bacteriology, vol. 1. The Williams \& Wilkins Co., Baltimore.

15. Jukes, T. H., and C. R. Cantor. 1969. Evolution of protein molecules, p. 21-132. In H. N. Munro (ed.), Mammalian protein metabolism. Academic Press, New York.

16. Laguerre, G., M. Bardin, and N. Amarger. Submitted for publication.

17. Laguerre, G., E. Geniaux, S. I. Mazurier, R. Rodriguez Casartelli, and N. Amarger. 1993. Conformity and diversity among field isolates of Rhizobium leguminosarum bv. viciae, bv. trifolii and bv. phaseoli revealed by DNA hybridization using chromosome and plasmid probes. Can. J. Microbiol. 39:412-419.

18. Lange, R. T. 1961. Nodule bacteria associated with the indigenous Leguminosae of South Western Australia. J. Gen. Microbiol. 26:351-359.

19. Lindström, K. 1989. Rhizobium galegae, a new species of legume root nodule bacteria. Int. J. Syst. Bacteriol. 39:365-367.

20. Maniatis, T., E. F. Fritsch, and J. Sambrook. 1982. Molecular cloning: a laboratory manual. Cold Spring Harbor Laboratory, Cold Spring Harbor, N.Y.

21. Martinez, E., M. Flores, S. Brom, D. Romero, G. Davila, and R. Palacios. 1988. Rhizobium phaseoli: a molecular genetics view. Plant Soil 108:179-184.

22. Martinez, E., M. A. Pardo, R. Palacios, and M. A. Cevallos. 1985. Reiteration of nitrogen fixation gene sequences and specificity to Rhizobium in nodulation and nitrogen fixation in Phaseolus vulgaris. J. Gen. Microbiol. 131:1779-1786.

23. Martinez-Romero, E., L. Segovia, F. M. Mercante, A. A. Franco, P. Graham, and M. A. Pardo. 1991. Rhizobium tropici, a novel species nodulating Phaseolus vulgaris L. beans and Leucaena sp. trees. Int. J. Syst. Bacteriol. 41:417-426.

24. Mazurier, S. I. 1989. Diversité de populations naturelles nodulantes de Rhizobium leguminosarum. Ph.D. thesis. Université Claude-Bernard Lyon I, Villeurbanne, France.

25. Murray, R. G. E., D. J. Brenner, R. R. Colwell, P. De Vos, M. Goodfellow, P. A. D. Grimont, N. Pfennig, E. Stackebrandt, and G. A. Zavarzin. 1990. Report of the Ad Hoc Committee on
Approaches to Taxonomy within the Proteobacteria. Int. J. Syst. Bacteriol. 40:213-215.

26. Nazaret, S., B. Cournoyer, P. Normand, and P. Simonet. 1991. Phylogenetic relationships among Frankia genomic species determined by use of amplified 16S rDNA sequences. J. Bacteriol. 173:4072-4078.

27. Pinero, D., E. Martinez, and R. K. Selander. 1988. Genetic diversity and relationships among isolates of Rhizobium leguminosarum biovar phaseoli. Appl. Environ. Microbiol. 54:28252832.

28. Quinto, C., H. de la Vega, M. Flores, L. Fernandez, T. Ballado, G. Soberon, and R. Palacios. 1982. Reiteration of nitrogen fixation gene sequences in Rhizobium phaseoli. Nature (London) 299:724-726.

29. Rigby, P. W. J., M. Dieckmann, C. Rhodes, and P. Berg. 1977. Labeling deoxyribonucleic acid to high specific activity in vitro by nick translation with DNA polymerase I. Int. J. Syst. Bacteriol. 113:237-251.

30. Roberts, G. P., W. T. Leps, L. E. Silver, and W. J. Brill. 1980. Use of two-dimensional polyacrylamide gel electrophoresis to identify and to classify Rhizobium strains. Appl. Environ. Microbiol. 39:326-333.

31. Sadowsky, M. J., P. B. Cregan, and H. H. Keyser. 1988. Nodulation and nitrogen fixation efficacy of Rhizobium fredii with Phaseolus vulgaris genotypes. Appl. Environ. Microbiol. 54:1907-1910.

32. Saitou, N., and M. Nei. 1987. The neighbor-joining method: a new method for reconstructing phylogenetic trees. Mol. Biol. Evol. 4:406-425.

33. Sanger, F., S. Nicklen, and A. R. Coulson. 1977. DNA sequencing with chain-terminating inhibitors. Proc. Natl. Acad. Sci. USA 74:5463-5467.

34. Scholla, M. H., and G. H. Elkan. 1984. Rhizobium fredii sp. nov., a fast-growing species that effectively nodulates soybeans. Int. J. Syst. Bacteriol. 34:484-486.

35. Segovia, L., D. Pinero, R. Palacios, and E. Martinez-Romero. 1991. Genetic structure of a soil population of nonsymbiotic Rhizobium leguminosarum. Appl. Environ. Microbiol. 57:426433.

36. Segovia, L., J. P. W. Young, and E. Martinez-Romero. 1993. Reclassification of American Rhizobium leguminosarum biovar phaseoli type I strains in a new species, Rhizobium etli sp. nov. Int. J. Syst. Bacteriol. 43:374-377.

37. Stackebrandt, E., R. G. E. Murray, and H. G. Trüper. 1988. Proteobacteria classis nov., a name for the phylogenetic taxon that includes the "purple bacteria and their relatives." Int. J. Syst. Bacteriol. 38:321-325.

38. Wayne, L. G., D. J. Brenner, R. R. Colwell, P. A. D. Grimont, O. Kandler, M. I. Krichevsky, L. H. Moore, W. E. C. Moore, R. G. E. Murray, E. Stackebrandt, M. P. Starr, and H. G. Trüper. 1987. Report of the Ad Hoc Committee on Reconciliation of Approaches to Bacterial Systematics. Int. J. Syst. Bacteriol. 37:463-464.

39. Willems, A., and M. D. Collins. 1993. Phylogenetic analysis of rhizobia and agrobacteria based on 16S rRNA gene sequences. Int. J. Syst. Bacteriol. 43:305-313.

40. Woese, C. R., E. Stackebrandt, W. G. Weisburg, B. J. Paster, M. T. Madigan, V. J. Fowler, C. M. Hahn, P. Blanz, R. Gupta, K. H. Nealson, and G. E. Fox. 1983. The phylogeny of purple bacteria: the alpha subdivision. Syst. Appl. Microbiol. 5:315326.

41. Young, J. P. W. 1985. Rhizobium population genetics: enzyme polymorphism in isolates from peas, clover, beans and lucerne grown at the same site. J. Gen. Microbiol. 131:2399-2408.

42. Young, J. P. W., H. L. Downer, and B. D. Eardly. 1991. Phylogeny of the phototrophic Rhizobium strain BTAil by polymerase chain reaction sequencing of a $16 \mathrm{~S}$ rRNA gene segment. J. Bacteriol. 173:2271-2277. 